\title{
Dinâmica de Populações, Modelo de Lotka-Volterra e Tecnologias: Análise de um Projeto Interdisciplinar
}

\author{
Ana Regina Gregory Brunet, ULBRA, FAPA, anabrunet@cpovo.net \\ Mariana de Souza Proença, PPGECIM - ULBRA, mariana.proenca@gmail.com \\ Marlise Geller, PPGECIM - ULBRA, marlise.geller@gmail.com \\ Rosangela Ferreira Prestes, URI, ro.fprestes@yahoo.com.br \\ Valmir Ninow, PPGECIM - ULBRA, vninow@gmail.com
}

Resumo: Esta pesquisa teve por objetivo analisar um projeto interdisciplinar envolvendo o uso do Facebook e do software Modellus como ferramentas de apoio ao ensino e aprendizagem nas áreas de Matemática e Biologia na disciplina de Matemática Aplicada da graduação em Matemática. A metodologia qualitativa aplicada envolveu quatro professores e seis alunos no estudo de modelos matemáticos sobre a dinâmica de populações no âmbito da ecologia. Percebe-se o impacto positivo do projeto, não somente nos alunos, mas na própria proposta da disciplina. A articulação entre a teoria e a prática, dada pela simulação a partir da busca de dados em artigos científicos para chegar aos parâmetros que foram construídos pelos alunos, proporcionou a compreensão do modelo em si, no caso específico, a relação presa/predador.

Palavras-chave: Projeto interdisciplinar; Facebook; Modellus; Educação Matemática; Matemática aplicada.

\section{Population Dynamics, Lotka-Volterra's Model and Technologies: Analysis of an Interdisciplinary Project}

\begin{abstract}
This research aimed to analyze an interdisciplinary project involving the use of Facebook and Modellus software as tools to support teaching and learning in the Biology and Mathematics areas in Mathematics Applied discipline of a Mathematics undergraduate course. The applied qualitative methodology involved four teachers and six students in the study of mathematical models of the dynamics of populations within the ecology. It is perceived the positive impact of the project, not only in the students, but in the proposed discipline intending to articulate the theory to practice through simulation from the data in search of scientific papers to arrive the parameters that were built by students, including the model itself, with specific regard to prey/predator.
\end{abstract}

Keywords: Interdisciplinary project; Facebook; Modellus; Mathematics Education; Applied Mathematics.

\section{Introdução}

O estudo da dinâmica de populações é importante para o entendimento do que ocorre nos ecossistemas em equilíbrio. População refere-se a um grupo de uma mesma espécie que se encontra em um mesmo habitat em um determinado espaço de tempo, podendo crescer ou diminuir. $\mathrm{O}$ crescimento de uma população depende de fatores como: natalidade, mortalidade, emigração, imigração, resistência ambiental.

Populações de duas espécies diferentes podem interagir de diversas formas, sendo essas interações chamadas de relações ecológicas interespecíficas. Essas relações podem ser consideradas harmônicas, quando há benefício para pelo menos uma das espécies, ou podem ser desarmônicas, a qual há prejuízo para uma ou ambas as espécies (ODUM, 1988). 
A predação é um exemplo de interação interespecífica que resulta em efeitos negativos no crescimento e sobrevivência de uma população e em um efeito positivo na outra. As equações de Lotka-Volterra retratam um sistema presa-predador de duas espécies onde uma espécie, o predador, determina a abundância da outra, a presa (AMAKU et al., 2007).

O estudo desses modelos matemáticos é de suma importância em conteúdo como a dinâmica de populações no âmbito da ecologia. Sendo assim observa-se a necessidade de se trabalhar interdisciplinarmente esse assunto.

Projetos Interdisciplinares é uma tendência na educação. As "relações entre conteúdos e áreas do conhecimento tem lugar em função das necessidades que traz consigo o fato de resolver uma série de problemas que subjazem na aprendizagem" o que para Hernández e Ventura (1998, p.63) seria a ideia fundamental dos projetos de trabalho.

Assim, como os projetos é uma tendência na educação, as tecnologias de informação e comunicação fazem parte da realidade do mundo atual. Mercado (1998, p.6) argumenta que "o objetivo de introduzir novas tecnologias na escola é para fazer coisas novas e pedagogicamente importantes que não se pode realizar de outras maneiras".

Esta pesquisa teve por objetivo analisar uma experiência em um projeto interdisciplinar envolvendo o uso de tecnologias como ferramenta de ensino e aprendizagem nas áreas de Matemática e Biologia, com o intuito de proporcionar aos acadêmicos de uma disciplina de Matemática Aplicada, da graduação em Matemática a vivência nesse projeto durante sua formação acadêmica.

A proposta do projeto consiste no estudo de Dinâmica de Populações a partir do modelo matemático de Lotka-Volterra com o apoio do software Modellus e a interação entre alunos e professores dinamizada em um grupo no Facebook. Em relação ao tema escolhido Peixoto et al (2004, p. 30) afirma que:

os modelos matemáticos que descrevem relações entre presa e predador são
utilizados para o estudo de interações que ocorrem entre duas populações nas
quais uma delas depende da outra para se alimentar e sobreviver. Tais
relações dinâmicas entre presa e predador são temas de destaque em ecologia.

O objetivo de utilizar o Modellus, nesse trabalho, é de possibilitar aos alunos a exploração das múltiplas representações presa/predador do modelo de Lokta-Voltera. O uso do Facebook teve por finalidade promover um ambiente interativo para os professores e alunos que desse suporte para a realização da atividade. O envolvimento dos estudantes na busca de informação para Hernández e Ventura (1998, p.75) "tem uma série de efeitos que se relacionam com a intenção educativa dos Projetos" dessa maneira enfatizando o papel do professor como facilitar nesse tipo de trabalho.

Com base no Hernández e Ventura (2003) foi elaborada a sequência de planejamento do Projeto. Os autores estabelecem três categorias de objetivos, a saber, conceituais, procedimentais e atitudinais.

Os objetivos conceituais elencados para o trabalho foram: o que e por que se estuda Dinâmica de Populações; compreender o conceito de população e também os conceitos que a envolvem; identificar as relações ecológicas existentes e identificar e classificá-las como harmônicas ou desarmônicas, intraespecífica ou interespecíficas; destacar e caracterizar a relação de Predação; caracterizar as espécies de par presa/predador (Joaninha/Pulgão) descrevendo seu habitat, modo de vida, reprodução; compreender a importância e a utilização dos meios virtuais de comunicação; compreender a importância e a utilização do modelo de Lotka-Volterra; compreender a 
contribuição de cada parâmetro do sistema de equações diferenciais do modelo de Lotka-Voltera; compreender a relação da Matemática com a modelagem e simulação de fenômenos da natureza.

Os objetivos procedimentais foram: aprender a buscar informações sobre determinado objeto em diferentes fontes; utilizar o Facebook como meio de suporte e interação; utilizar o Modellus para simulação de modelos de fenômenos; utilizar conceitos biológicos para simular a relação ecológica presa/predador com o uso do modelo de Lotka-Volterra.

Por fim, os objetivos atitudinais almejados foram: refletir como utilizar diferentes tecnologias como ferramenta didática para os processos de ensino e aprendizagem; perceber a importância das relações ecológicas na dinâmica de populações; utilizar o Modellus como ferramenta de apoio em simulações de fenômenos; tomar consciência da importância de trabalhar interdisciplinarmente; perceber e utilizar a Matemática para descrever fenômenos da natureza.

A proposta interdisciplinar de certa maneira propõe uma organização das informações, dos conhecimentos com base em uma visão disciplinar centralizando-se em alguns temas contemplado por diversos métodos e finalidades. Para Fazenda (2008, p.9) "a interdisciplinaridade se consolida na ousadia da busca, de uma busca que é sempre pergunta, ou melhor, pesquisa".

Segundo Hernández e Ventura (2003, p.61) a função dos projetos seria de "favorecer a criação de estratégias de organização dos conhecimentos escolares" com intuito de facilitar "a construção de seus conhecimentos e a transformação da informação procedente dos diferentes saberes disciplinares em conhecimento próprio", desse modo os Projetos estão vinculados a aprendizagem globalizadora. Na concepção de Fazenda (1991, p.31) a interdisciplinaridade "caracteriza-se pela intensidade das trocas entre os especialistas e pela integração das disciplinas num mesmo projeto de pesquisa".

As Tecnologias de Informação e Comunicação vem causando grandes impactos na sociedade. Os recursos escolhidos para o trabalho foram o software Modellus e a rede social Facebook.

O Modellus é um software que permite a simulação de modelos de fenômenos físicos, químicos, econômicos e do cotidiano de uma maneira geral utilizando equações matemáticas que as representam. Dessa forma, o usuário introduz o modelo matemático e o software realiza a simulação computacional. No programa, também, podem ser controladas as variáveis como: o tempo, a distância e a velocidade. Desta forma, o programa permite analisar a variação das funções graficamente, preparar animações, resolver exercícios e criar seus próprios exercícios conforme a necessidade.

Sobre as vantagens no uso do Modellus, Ribeiro et al (2005) destacam a facilidade do aluno em poder variar os parâmetros envolvidos nos modelos e conseguir uma saída gráfica, além de animação e ferramentas de medição. Desta forma, num único ambiente, eles poderão apresentar o mesmo objeto sob diferentes perspectivas: fórmulas, gráficos, tabelas, vetores e animações. Permitindo, dessa maneira, testar e manipular os diferentes dados levantados na pesquisa sobre o tema presa/predador (Dinâmica de Populações), bem como, oportunizar aos alunos a capacidade de apresentar e manipular visões diferentes e complementares de uma mesma ideia.

Em relação ao Facebook, é uma das redes sociais que, atualmente, está sendo utilizada em larga escala como espaço para encontro de amigos, compartilhamento de informações, interações e discussões de ideias dos mais diferentes temas. O Facebook é um ambiente criado para que qualquer indivíduo tenha a liberdade de se expressar, comunicar, compartilhar e interagir. Em relação a estes aspectos mencionados, pode-se 
dizer que o seu objetivo foi atingido, pois cada vez mais cresce o número de pessoas que passaram a utilizar esta rede social. Além ser uma rede social gratuita, o Facebook apresenta um conjunto de funcionalidades comuns a outras redes sociais e também a capacidade de agregar conteúdos de outros locais da Web.

\section{Metodologia}

O público alvo constituiu-se em acadêmicos de graduação em Licenciatura em Matemática e Bacharelado em Matemática Aplicado a Informática de uma disciplina de Matemática Aplicada, cujo conteúdo versa principalmente sobre equações diferenciais na qual a contextualização das ferramentas matemáticas desenvolvidas são ilustrativas e necessárias. Desta maneira, foi proposta uma contextualização dos modelos matemáticos na área de Biologia, com enfoque na ecologia de populações para uma atividade semipresencial já prevista no cronograma da disciplina.

Para viabilizar a execução da pesquisa três professores de Matemática e um de Biologia atuaram em parceria ao longo de quatro etapas: elaborar uma atividade interdisciplinar envolvendo conceitos matemáticos e biológicos com o uso de tecnologias; promover um ambiente de interação e suporte através da rede social Facebook; construir um tutorial do software Modellus e avaliar o desenvolvimento do projeto a partir do material coletado tanto pela atividade realizada pelos alunos como por um questionário avaliativo respondido por eles. A seguir, descreve-se brevemente as etapas citadas.

A primeira atividade do grupo de professores correspondeu à elaboração de uma atividade interdisciplinar envolvendo conceitos matemáticos e biológicos com o uso de tecnologias. Os recursos tecnológicos utilizados nessa proposta correspondem a um grupo no Facebook e o programa Modellus.

O Facebook foi selecionado com a finalidade de construir um grupo particular para o acesso e contato virtual dos alunos e professores durante a realização da atividade. Incialmente foi construído um grupo no Facebook intitulado Tecnologia de Informação e Comunicação com acesso somente aos professores para sua organização e troca de informações durante todo o processo de atividade.

A partir de uma pesquisa prévia, em sala de aula, constatou-se que alguns alunos não tinham conta no Facebook. Sendo assim, organizou-se um tutorial contendo informações básicas e necessárias para o acesso a essa rede social o qual foi disponibilizado aos alunos por email pela professora responsável pelo ambiente, com quem as dúvidas poderiam ser discutidas. O grupo intitulado Dinâmica de Populações foi utilizado para fins de orientação durante o trabalho. Também foi possível esclarecer dúvidas presencialmente no horário da disciplina.

Simultaneamente, foi construído um tutorial do software Modellus, com intuito de ser disponibilizado no grupo do Facebook, para ser utilizado durante a realização das tarefas propostas. O tutorial forneceu o passo a passo para o download do programa e informações básicas do seu uso. Qualquer dúvida sobre o Modellus poderia ser tratada via Facebook com o professor responsável pelo software.

A atividade proposta foi desenvolvida em duas fases. Na primeira fase da atividade o aluno teve que pesquisar e discutir sobre Dinâmica de Populações, população, relações ecológicas interespecíficas, predação e, além disso, sobre o modelo de Lotka-Volterra. Foram sugeridas, pelos professores, algumas bibliografias. Na segunda fase, o par presa/predador foi determinado pelos professores: pugões/joaninhas. Os alunos deveriam pesquisar sobre estas determinadas espécies, descrevendo seu habitat, modo de vida, reprodução entre outras coisas que julgassem interessantes a 
respeito do par. Para essas informações os estudantes puderam solicitar auxílio para a professora de Biologia via grupo Dinâmica de Populações no Facebook.

Após a pesquisa realizada os alunos foram desafiados, na etapa seguinte, a simular a relação pulgão/joaninha no modelo de Lokta-Voltera, utilizando como apoio o software Modellus, conforme pode ser visto na figura 1. Para tanto, inicialmente foram sugeridas a variação dos parâmetros e condições iniciais a fim de investigar a contribuição de cada um no sistema de equações do modelo de Lokta-Voltera. A seguir, conscientes do modelo e embasados na pesquisa, pediu-se o sistema (parâmetros) que melhor representassem a relação do par pulgão/joaninha.

Figura 1 - Simulação da relação pulgão/joaninha

No gráfico 1 (Imagem 4), com $\mathrm{x}=0.5$ e $\mathrm{y}=0.01$, representa os seguintes parâmetros: $\mathrm{A}=1 ; \mathrm{B}=3 ; \mathrm{C}=4 ; \mathrm{D}=5$.

Com esses parâmetros, no meu ponto de vista, seria o ideal, pois temos sempre mais pulgões do que joaninhas e assim tendo sempre um equilíbrio ambiental, pelo fato de que cada joaninha come em média 100 pulgões por dia.

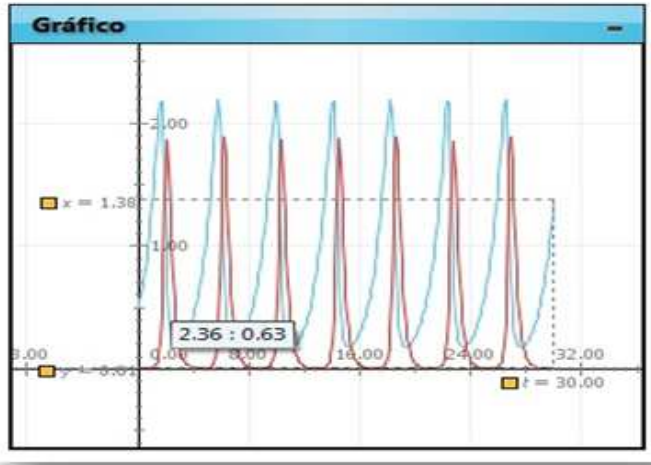

Imagem 4 - Gráfico 1

Fonte: A pesquisa

Convém observar que foi solicitado aos alunos o registro das diferentes simulações pensadas e justificativas do por que das novas atribuições aos parâmetros.

Figura 2 - Simulações - variações dos parâmetros

O gráfico 2 (Imagem 5), representa os seguintes parâmetros: $A=1 ; B=2 ; C=3 ; D=4$.

Com esses parâmetros a quantidade de joaninhas é maior que a de pulgões pelo fato da taxe de crescimento das presas ter diminuido em relação ao gráfico anterior, no meu entendimento isso traria um desequilibrio ambiental, pois teriamos muito mais predadores e consequentemente sua morte por falta de presas.

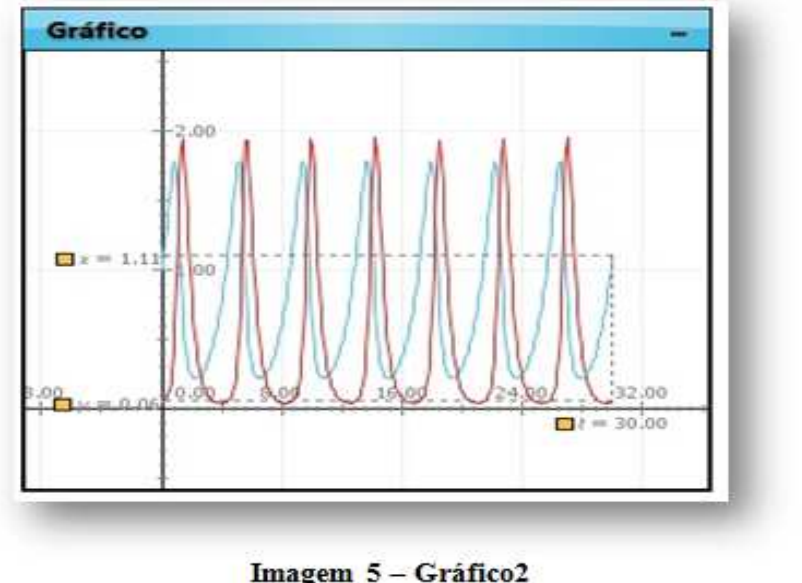

Imagem 5 - Gráfico2

Fonte: A pesquisa 
Na proposta de atividade, encaminhada aos alunos via Facebook, constaram informações como a data prevista para entrega do trabalho digitado. Esse trabalho tinha que conter capa, introdução incluindo a pesquisa sobre Dinâmica de Populações e modelo de Lotka-Volterra, desenvolvimento com registro das atividades propostas com o par presa/predador, considerações finais - entre as quais um parecer do aluno a respeito da experiência interdisciplinar proposta - e as referências utilizadas.

$\mathrm{O}$ anteprojeto foi apresentado oralmente à direção da instituição, representada pelo coordenador do curso de Matemática. Após aprovação, o coordenador foi adicionado como membro do grupo Dinâmicas de População.

Para avaliação do Projeto Interdisciplinar foram coletadas informações na atividade entregue (a opinião de cada estudante sobre a importância de atividades interdisciplinares para sua formação solicitada na conclusão da atividade), um parecer do grupo de professores envolvidos diretamente no projeto e um parecer do coordenador do curso.

\section{Análise e discussão dos resultados}

Dos seis alunos definidos como amostra que realizaram o trabalho da disciplina, cinco responderam o questionário aplicado após a atividade. Desses cinco alunos, foi traçada a média de idade entre eles, sendo 28 anos, e a percepção referente ao trabalho proposto.

Quatro desses alunos já tiveram em sua vida escolar/acadêmica contato com algum tipo de tecnologia da comunicação, como disciplinas a distância e apenas um utilizou software relacionado à disciplina de matemática. Já em relação à rede social Facebook apenas dois alunos a utilizavam para comunicação com amigos e familiares e um também para fins comerciais. Nenhum dos estudantes conhecia o software Modellus.

Todos os participantes consideraram de grande utilidade o Modellus. Registraram que "proporcionou uma melhor visualização" do fenômeno estudado "com mais ideias e certezas do assunto". Um facilitador para a simulação "com ele consegui ir mudando os números [parâmetros] e analisando o gráfico ao mesmo tempo" dando "sentido a pesquisa feita [...] visualizar o comportamento que cada espécie apresentou no gráfico". Porém, "...muita dificuldade de usar o Modellus", "levou bastante tempo para atinarmos a mexer no Modellus" e "difícil de usar" indicam a dificuldade encontrada, mas superada, pelos alunos ao interagir com o software.

O Facebook foi apontado como facilitador na comunicação entre colegas e professores. Colocações como "ocorreram várias trocas de ideias, informações, esclarecimentos de dúvidas", "conversando com os colegas, trocando informações, pedindo ajuda foi muito mais fácil concluir o trabalho" e "as dúvidas apareciam e eram esclarecidas ali mesmo, naquele momento" são registros que ratificam a colocação acima. Além disso, "pela acessibilidade com os professores de diversas áreas e com os colegas" o Facebook mostrou-se uma novidade pedagogicamente importante, "esta ferramenta foi de suma importância para a realização deste trabalho". "Tínhamos apenas um encontrou por semana" e "sem o facebook acho que não teria trabalho algum" sugerem que a rede social foi determinante para o bom andamento do trabalho. Desta forma, cumpriu-se o objetivo de Mercado (1998, p. 6) ao utilizar recursos para efetivar o trabalho de forma distinta de uma aula somente presencial.

Em relação às duas tecnologias utilizadas no trabalho, a opinião dos estudantes foi muito satisfatória, destacando, novamente, a agilidade de suporte e interação proporcionada pelo Facebook e a melhor compreensão do fenômeno estudado através 
das ferramentas do Modellus. Como exemplo de resposta satisfatória, constata-se: "qualquer rede social ou software atribuídos a um trabalho fica bem mais interessante e fácil de ser realizado".

Um dos objetivos do trabalho era propiciar a vivência de um projeto interdisciplinar para fomentar a futura prática profissional dos estudantes envolvidos. Sendo assim, em relação à relevância da temática Dinâmica de Populações proposta no projeto os participantes declararam "interessante" pela integração de outras áreas de conhecimento com a Matemática. Isso é percebido nos registros: "pesquisamos assuntos diferentes e interligamos ao conteúdo da Matemática" e "através de todas as etapas do trabalho tivemos a oportunidade de vivenciar como funciona um trabalho interdisciplinar".

Em relação à escolha da temática Simulação de Modelos Matemáticos expressos em Equações Diferenciais, todos os alunos manifestaram-se positivamente, expressando que "a simulação do modelo foi de grande valia para entendermos como funciona a modelagem" e "com este trabalho tivemos a oportunidade de aplicar uma modelagem, que tem tudo a ver com a disciplina que estamos cursando...".

A professora da turma havia manifestado interesse de "mudança no desenrolar da disciplina [...] algo que associasse as outras áreas do conhecimento de maneira significativa para os alunos". Sua intenção com o projeto interdisciplinar realizado vai ao encontro das ideias de Hernández e Ventura (2003), pois essas propostas não podem ser fragmentadas, se limitando ao tema a ser trabalhado separadamente em cada disciplina, ao contrário, devem proporcionar uma aprendizagem globalizadora, estabelecendo vínculos entre diferentes fontes de informação.

No seu parecer o coordenador coloca que achou "a proposta muito interessante, pois proporcionou aos alunos da disciplina de Matemática Aplicada uma interação interdisciplinar, fazendo com que os mesmos pudessem ver uma utilização prática do conteúdo estudado na disciplina", também corrobora com as ideias de Hernández e Ventura (2003). Além disso, "outro aspecto importante foi o trabalho de pesquisa que os alunos tiveram que realizar". Ainda em seu parecer, o coordenador do curso apoia e parabeniza "a iniciativa e a proposta do grupo de professores envolvidos, pois, acredito, fez com que possamos pensar diferente em relação à metodologia das nossas disciplinas do Curso de Matemática".

O êxito da atividade proposta também pode ser verificado nos registros solicitados em parágrafo do trabalho escrito e entregue pelos alunos via Facebook, como ilustrado pelo comentário a seguir:

Essa experiência gerou grandes aprendizagens, com a inclusão do software Modellus na área da matemática com a junção da Biologia, isso na área da aprendizagem é algo fundamental, permite-nos aprender que existem outras ferramentas e tecnologias para interagir com várias disciplinas e conteúdos, como no trabalho que estou apresentando, esse trabalho foi muito proveitoso e motivador pra toda a turma de aplicada.

Ainda observaram que "o trabalho proposto pelo grupo de professores foi de grande valor [...] além de proporcionar uma experiência interdisciplinar nos fez buscar conceitos em diferentes áreas".

Sobre a área da Biologia escolhida, destaca-se o registro de um aluno:

o estudo sobre as relações ecológicas teve com principal objetivo a percepção da dinâmica entre os seres vivos de um ambiente. É importante compreender as relações existentes entre os seres de espécies diferentes e da mesma espécie, e como essas relações colaboram para o equilíbrio ecológico 
e que o trabalho "fez buscar conceitos em diferentes áreas". Estas impressões evidenciam o envolvimento dos alunos com o projeto.

\section{Considerações finais}

Embora a interdisciplinaridade do projeto seja caracterizada pelo olhar da Matemática sobre a Biologia o êxito do trabalho ocorreu devido o intermédio da tecnologia representado pelo Facebook e o Modellus.

Percebe-se o impacto positivo do projeto, não somente nos alunos da disciplina que participaram efetivamente da experiência, mas na própria proposta da disciplina de Matemática Aplicada objetivando articular a teoria à prática. A essa articulação ocorreu através da simulação a partir da busca de dados em artigos científicos para chegar aos parâmetros que foram construídos pelos alunos, compreendendo o modelo em si, no caso específico a relação presa/predador (pulgão/joaninha).

Acreditamos que pouca interação com software educacional verificada nos registros, explica, em parte, a dificuldade encontrada pelos estudantes no funcionamento do Modellus.

\section{Referências}

AMAKU, M.; FERREIRA, F.; OLIVEIRA, D.F. Produção de material didático para o ensino de dinâmica de populações. Universidade São Paulo. 20/08/2007. Disponível em: http://vps.fmvZ.usp.br/promat/lotkavolterra.html. Acesso em 30 jun. 2012.

HERNÁNDEZ, F. e VENTURA, M. A organização do currículo por projetos de trabalho. Porto Alegre: Artes Médicas, 1998.

HERNANDEZ, F; VENTURA, C. B. Transgressão e mudança na educação. Porto Alegre: Artmed, 2003.FAZENDA, I.C.A. Interdisciplinaridade: história, teoria e pesquisa. 15 ed. Campinas: Papirus, 2008.

1991.

Interdisciplinaridade: um projeto em parceria. São Paulo: Loyola,

MERCADO, L. P. L. Formação docente e novas tecnologias. In: IV Congresso RIBIE. Brasilia, $1998 . \quad$ Disponível em: http://lsm.dei.uc.pt/ribie/docfiles/txt200342414941210M.PDF. Acesso em 30 jun. 2012. MINHOTO, Paula; MEIRINHOS, Manuel. O facebook como plataforma de suporte à aprendizagem da Biologia. In: Conferência Ibérica: Inovação na Educação com TIC. Bragança: Instituto Politécnico de Bragança, 2011. p. 118-134. ISBN 978-972-745-1203. Disponível em: http://bibliotecadigital.ipb.pt/handle/10198/6179. Acesso em 30 jun. 2012.

ODUM, Eugene P. Ecologia. Rio de Janeiro: Guanabara, 1988.

PEIXOTO, M.S., BARROS, L.C. e BASSANEZI. Um modelo de Fuzzy PresaPredador em Citros: pulgões e joaninhas. BIOMATEMÁTICA 14. Campinas, 2004. p. 29-38.

RIBEIRO, Y.H.L., JESUS, J.C.O. e ALVES, A.S. Utilização do Modellus na construção de conceitos físicos. Anais do XVI Simpósio Nacional de Ensino de Física. Rio de Janeiro, 2005. 